\title{
Genetic polymorphisms of CYP2D6 oxidation in patients with autoimmune bullous diseases
}

\author{
Mariola Rychlik-Sych ${ }^{1}$, Małgorzata Baranska1, Elzbieta Waszczykowska², Jolanta Dorota Torzecka², \\ Agnieszka Zebrowska ${ }^{2}$, Jadwiga Skretkowicz ${ }^{1}$
}

1Department of Pharmacogenetics, Medical University of Lodz, Poland

Head: Prof. Jadwiga Skretkowicz

2Department of Dermatology and Venereology, Medical University of Lodz, Poland

Head: Prof. Anna Sysa-Jedrzejowska MD, PhD

Postep Derm Alergol 2013; XXX, 4: 211-217

DOI: 10.5114/pdia.2013.37030

\begin{abstract}
Introduction: Bullous skin diseases, which include, among others pemphigoid, pemphigus, and dermatitis herpetiformis are classified as severe autoimmune dermatoses. It has been shown that a pattern of xenobiotic metabolism may play a role in the pathogenesis of autoimmune diseases.

Aim: To estimate whether the CYP2D6 genotype may be considered a predisposing factor in autoimmune bullous diseases induction.

Material and methods: The study included 72 patients with autoimmune bullous diseases: 37 with pemphigoid, 21 with pemphigus, and 14 with dermatitis herpetiformis (DH). The CYP2D6 genotypes were analyzed by the polymerase chain reaction fragment length polymorphism (PCR-RFLP) method.

Results: Relative risk of DH development for particular genotype carriers expressed by odds ratio (OR) was statistically significantly higher for subjects with CYP2D6*1/CYP2D6 $6^{\star} 4(\mathrm{OR}=4.2 ; p=0.0104)$ and 2-fold higher for subjects with CYP2D6*4 (OR $=2.3 ; p=0.0351)$.

Conclusions: The results of the present study show that the CYP2D6 oxidation polymorphism cannot be considered a risk factor for development of pemphigoid and pemphigus, however it might have an impact on dermatitis herpetiformis.
\end{abstract}

Key words: genetic polymorphism, CYP2D6, pemphigoid, pemphigus, dermatitis herpetiformis.

\section{Introduction}

Bullous skin diseases, including pemphigoid, pemphigus, and dermatitis herpetiformis are considered severe autoimmune dermatoses. These diseases are characterized by bullae and bullous eruptions on the skin and within the mucosa. Depending on the site of bulla formation in the epidermis, bullous diseases are divided into intraepidermal (acantholytic), which comprise all types of pemphigus, and subepidermal ones including pemphigoid and dermatitis herpetiformis [1-3].

Pemphigoid (bullous pemphigoid - BP) is the most frequently occurring entity among bullous diseases. It develops mainly in the elderly people, and the relative risk of its prevalence at the age of 90 years is nearly 300 -fold higher than at the age of 60 [4].
Genetic involvement in pemphigoid has not been clarified so far. The association with HLA DR4, DR5, and DQw3 antigens implies a role of genetic factors in etiopathogenesis of this disease. Pemphigoid may be also induced by orally administered drugs, e.g. furosemide or novoscabin applied topically to the skin $[1,5]$.

Pemphigus occurs in two basic forms: pemphigus vulgaris (PV) and pemphigus foliaceus (PF), which differ in the clinical picture and depth of acantholysis formation (damage to the connections between keratinocytes). Pemphigus belongs to genetically conditioned diseases with a dominating way of inheritance. There are some reports on familial prevalence of this disease and the relationship with $\mathrm{HLA} \mathrm{DR}^{\star} 4$, DRw6, and DQw8. Cases of pemphigus concomitant with other autoimmune diseases, e.g. rheumatoid arthritis, sys-

Address for correspondence: Agnieszka Zebrowska MD, PhD, Department of Dermatology and Venereology, Medical University of Lodz, 5 Krzemieniecka St, 94-017 Lodz, Poland, e-mail: agnieszka.zebrowska@umed.lodz.pl

Received: 9.11.2012, accepted: 29.04.2013. 
temic lupus erythematosus, or pemphigoid, have been observed [6-9]. Moreover, effects of environmental factors on pemphigus development have been also considered. In some cases, drugs (penicillin, captopril, butapirazol), sunlight, skin burns, viral (Herpetoviridae family) infections and some foods, e.g. garlic, leek, onion (containing a thiol group responsible for induction of chemical acantholysis) may become diseaseprovoking factors [1, 9].

Dermatitis herpetiformis (DH) is a chronic bullous disease. Due to concomitance with intestinal hypersensitivity, Duhring's disease is classified as cutano-intestinal syndrome. It most often afflicts young white men and children. Familial predisposition to DH has been emphasized; siblings more often suffer from this disease than parents and children. In DH pathogenesis, the relationship with HLAB8, DR3, DQW2, and DR2 is frequently mentioned $[1,10-12]$.

Bullous skin diseases develop as a result of disorders of the tolerance to self-antigens. Acantholytic bullous dermatoses are characterized by the presence of antibodies directed against desmosomal structures (bridges connecting keratinocytes) and surface antigens of keratinocytes, whereas subepidermal bullous dermatoses induce antigens against semidesmosomal/hemidesmosomal structures and the basement membrane [2].

The pathomechanism of developing cutaneous lesions in the course of bullous skin diseases has not been completely known so far. Similar to other autoimmune diseases, the role of genetic and environmental factors has been emphasized in etiopathogenesis of bullous diseases. The genetic factor involvement has been implied by their higher prevalence among patients' relatives, the relationships with HLA alleles, tissue compatibility antigens and polymorphism of numerous genes coding complement constituents, some receptors and cytokines.

Due to the significant role that environmental factors play in the pathogenesis of autoimmune diseases, various studies have been carried out to explain the way in which xenobiotics may affect the occurrence of autoimmune processes leading to the development of these diseases. Many xenobiotics such as drugs, pesticides, fungicides or substances that cause environmental pollution may induce changes in the chromosomes structure, leading to genetic mutations [13-15]. Xenobiotics involved in initiation of pathological processes of autoimmune diseases, similarly to all xenobiotics, undergo metabolic processes in the human body.

Among numerous enzymes involved in xenobiotic metabolism, the enzymatic system of hepatic microsomal fraction, the so-called monooxygenase system (multifunctional monooxygenase, mixed function oxygenaseMFO), whose main component is cytochrome P-450 enzymes, is responsible for oxidation processes belonging to phase I metabolism. Currently, the role of cytochrome P-450 and its isoenzymes have been considered significant both in regulating drug metabolism and determining other functions of the organism, such as inducing the immunological response or the processes, which are responsible for activation of toxic substances.

Cytochrome P-450 enzymes demonstrate the genetic polymorphism caused by the presence of mutation in the gene responsible for synthesis of a particular enzymatic protein. The mutation occurrence leads to individual variability of isoenzyme metabolic activity manifesting through a lack, reduction, or increased activity of the enzyme.

CYP2D6, called debrisoquine hydroxylase, is one of the best-known significant clinical cytochrome P-450 isoenzymes manifesting genetic variability. The gene responsible for the expression of CYP2D6 enzyme is located on chromosome 22q13.1 [16, 17]. Approximately 100 CYP2D6 alleles have been identified, among which over 20 significantly change metabolism of drugs that are substrates of this enzyme $[18,19]$. Depending on the metabolic rate, a human population has been divided into: extensive metabolizers (EM), ultrarapid metabolizers (UM), intermediate metabolizers (IM), and poor metabolizers (PM). Poor metabolizers genotype corresponds to a homozygous genotype of two recessive genes responsible for impaired metabolism, while extensive metabolizers of xenobiotics may be homozygotes carrying two genes conditioning fast oxidation phenotype or heterozygotes possessing one gene responsible for slow, and another one for fast oxidation. Ultrarapid metabolizers additionally have a greater number of active genes at the same locus [16, 19].

The studies assessing susceptibility to develop diseases in extensive metabolizers indicated a higher prevalence of pulmonary and genitourinary neoplasms as well as acute myeloblastic leukemia in these subjects. While among poor metabolizers, an increase in the prevalence of Parkinson's disease and epilepsy was observed; however, the results of different groups of researchers are not coherent [20-25]. Literature reports on the relationship between CYP2D6 fast oxidation and the prevalence of various diseases, mostly neoplastic and neurological, have encouraged researchers to look for such relations also in dermatological diseases.

Recently, an interest in the role of polymorphism of enzymes metabolizing xenobiotics in systemic lupus erythematosus (SLE) and systemic sclerosis (SSC), regarded as autoimmune diseases, has risen [26-36]. However, reports on the polymorphism of enzymes metabolizing xenobiotics in bullous skin diseases are scarce and mostly concern the genetic polymorphism of thiopurine methyltransferase (TPMT) and N-acetyltransferase 2 (NAT2) involved in the metabolism of drugs administered in the treatment of these diseases [37-41].

An attempt was made in the present study to assess genetically determined polymorphism of CYP2D6, which is one of the isoenzymes mediating phase I drug metabolism in patients suffering from skin bullous diseases. Moreover, the usefulness of CYP2D6 genotype investigation and the prevalence of CYP2D6 gene mutation were determined to evaluate susceptibility to bullous disease development, 
including pemphigoid, pemphigus, and dermatitis herpetiformis.

\section{Material and methods}

The study comprised 72 patients with skin bullous diseases: 37 with pemphigoid, 21 with pemphigus, and 14 with dermatitis herpetiformis. There were 40 women and 32 men aged 19-92 years (mean age 60.6 \pm 21.36 years). All patients were treated at the Department of Dermatology and Venereology at the Medical University of Lodz.

Pemphigoid was diagnosed based on the clinical picture, and histological and immunological findings. The patients were at an active stage of the disease, 30 out of 37 patients were presented with skin blisters, vesicles and itching papules, whereas others had only small vesicles and urticarial papules. In all the patients, the direct immunofluorescence test revealed lgG/C3 linear deposits along BMZ. In the salt split test, deposits were observed in the epidermal part of the blister. Using the indirect immunofluorescence test, circulating IgG antibodies were found in 33/37 patients. Whereas the ELISA test showed the anti-Nc16 autoantibodies (MBL, Nagoya, Japan) present in serum of 35 out of 37 patients. Typical histological features of BP including neutrophilic infiltrates, eosinophils, lymphocytes and in 35 cases - subepidermal blisters supported the clinical diagnosis.

Pemphigus was diagnosed based on the clinical picture, and histological and immunological findings. The patients were at an active stage of the disease, 18 out of 21 patients were presented with skin blisters, vesicles and erythema, whereas others had only small vesicles and erosions. In all the patients, the direct immunofluorescence test revealed IgG/C3 deposits form a network-like pattern. Using the indirect immunofluorescence test, circulating pemphigus antibodies were found in 19/21 patients.

The study included untreated patients with $\mathrm{DH}$ in an active stage of the disease. Ten out of 14 patients had skin lesions characterized by vesicles and itching papules, the others had erythematous papules. In all cases histological pictures showed perivascular neutrophilic infiltrates, the presence of Pierrard's abscesses, and in all patients small subepidermal blisters. In 10/14 samples, large unilocular blisters displaying multiple neutrophilic papillary microabscesses were found. Direct immunofluorescence tests revealed the presence of granular deposits of IgA in skin papillae and indirect immunofluorescence tests were positive for IgAEmA (Oesophagus monkey IgAEmA, Medizinische Labordiagnostica) in all the patients (titer $1: 10$ -1 : 320, median 1 : 40). Anti-tissue transglutaminase antibodies measured using an immunoassay (Celikey, Pharmacia \& Upjohn) were present in 6/14 cases (median $4.3 \mathrm{lU} / \mathrm{ml}$, range: $0.0-156.3 \mathrm{lU} / \mathrm{ml}$ ). Diagnosis of $\mathrm{DH}$ was established based on clinical presentation and results of histological and immunological examinations.

The control group included 129 healthy volunteers, 80 women and 49 men aged between 18 and 73 years (mean age $41.3 \pm 15.41$ ). The study was approved by the University Bioethics Committee for Research on Human Subjects.

Identification of the CYP2D6 gene alleles (CYP2D $6^{\star} 1$, CYP2D6*3, CYP2D6*4) was performed by using the PCRRFLP method according to the procedure described by Smith et al. [42]. Genomic DNA isolated from peripheral blood leukocytes according to Gustincich et al. [43] was amplified using the PCR method with a pair of synthetic oligonucleotides, separately for CYP2D $6^{\star} 3$ and CYP2D $6^{\star} 4 \mathrm{mu}$ tations. To detect the presence or absence of mutations at position A1934T (CYP2D6*4 mutation) and deletions at position A2637 (CYP2D6*3 mutation), the obtained product of PCR amplification was subjected to digestion by restrictive enzymes BstN1 (New England BioLabs, USA) (CYP2D6*4 mutation) and Hpa II (Invitrogen USA) (CYP2D6*3 mutation). The separation of digestion products was carried out in $8 \%$ polyacrylamide gel. The received stripes reflecting particular DNA fragments were visualized in UV light after ethidium bromide staining.

\section{Statistical analysis}

Statistical analysis was performed by using the $\chi^{2}$ test. A relative risk of disease development was determined by calculating odds ratio (OR) with the appropriate confidence level and the use of Statistica 9.1 software.

\section{Results}

The study results indicated that the frequency distribution of extensive and poor metabolizers in the group of

Table 1. Frequency distribution of poor and extensive metabolizers in patients with pemphigoid (BP), pemphigus and dermatitis herpetiformis $(\mathrm{DH})$ and in the controls

\begin{tabular}{|c|c|c|c|c|c|c|c|c|c|c|c|c|c|}
\hline \multirow{2}{*}{$\begin{array}{l}\text { Oxidation } \\
\text { genotype }\end{array}$} & \multirow{2}{*}{$\begin{array}{c}\text { Control } \\
\text { group } \\
(N=129) \\
n(\%)\end{array}$} & \multicolumn{3}{|c|}{ Bullous diseases $(N=72)$} & \multicolumn{3}{|c|}{$\mathrm{BP}(N=37)$} & \multicolumn{3}{|c|}{ Pemphigus $(N=21)$} & \multicolumn{3}{|c|}{$\mathrm{DH}(N=14)$} \\
\hline & & $n(\%)$ & $x^{2}$ & $p$ & $n(\%)$ & $x^{2}$ & $p$ & $n(\%)$ & $x^{2}$ & $p$ & $n(\%)$ & $x^{2}$ & $p$ \\
\hline Poor & $12(9.3)$ & $6(8.3)$ & 0.05 & 0.8176 & $4(10.8)$ & 0.0017 & 0.9666 & 0 & 1.05 & 0.3061 & $2(14.3)$ & 0.02 & 0.9025 \\
\hline Extensive & $117(90.7)$ & $66(91.7)$ & & & $33(89.2)$ & & & $21(100)$ & & & $12(85.7)$ & & \\
\hline
\end{tabular}

$p$-significantly lower $(p<0.05), \chi^{2}-$ test comparing two groups 

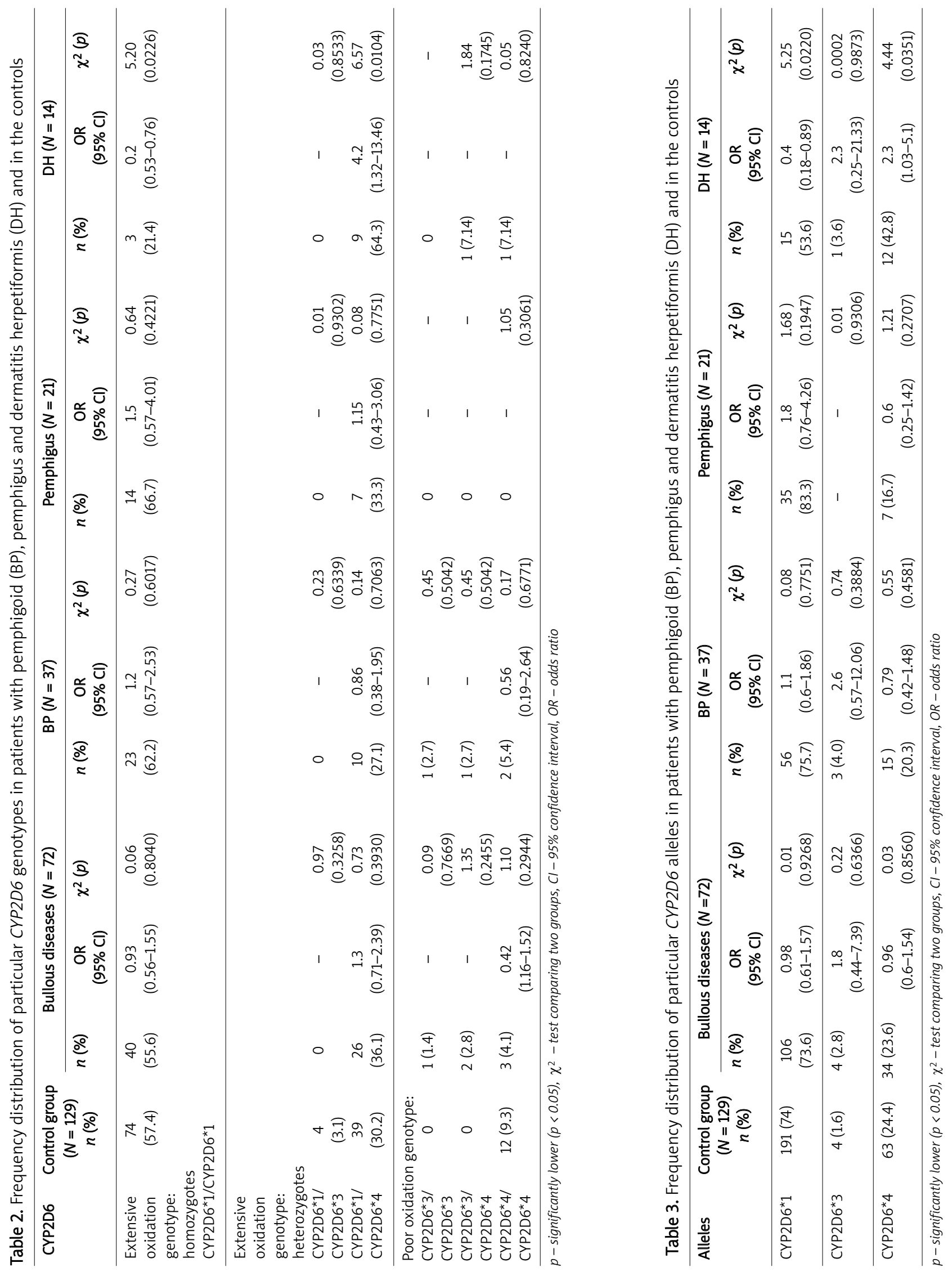
patients suffering from bullous skin diseases including pemphigoid, pemphigus, and dermatitis herpetiformis was similar to the control group (Table 1).

Table 2 shows the results of examination of CYP2D6 genotype in patients with bullous skin diseases. The CYP2D $6^{\star} 1 / C Y P 2 D 6^{\star} 1$ genotype was most frequently found among the patients with bullous diseases including pemphigoid and pemphigus as well as in the control group (55.6\%, 62.2\%, 66.7\%, and 57.4\%, respectively). However, in the group of patients with dermatitis herpetiformis, the percentage of the dominating homozygotes was lower (21.4\%) than in the control group (57.4\%). The differences were statistically significant $(p=0.0226)$. The patients with dermatitis herpetiformis most frequently had the CYP2D6*1/CYP2D6*4 genotype (64.3\%), whereas in the control group its prevalence was $30.2 \%$; the differences being statistically significant $(p=0.0104)$. The relative risk of developing dermatitis herpetiformis expressed by the odds ratio was over 4 times higher for subjects with the CYP2D6*1/CYP2D6*4 genotype $(\mathrm{OR}=4.2 ; p=0.0104)$.

Based on the results of genotyping, the frequency distribution of particular alleles of the CYP2D6 gene was assessed in the group of patients with bullous skin diseases including pemphigoid, pemphigus, and dermatitis herpetiformis, as well as in the controls (Table 3 ). In all groups of patients as well as in the control group, the wild-type CYP2D6 ${ }^{\star} 1$ allele was most common (73.6\%, 75.7\%, 83.3\%, $53.6 \%, 74.0 \%$, respectively). The variant wild-type CYP2D6* allele occurred more rarely only in the patients with dermatitis herpetiformis (53.6\%) as compared to the controls (74.0\%); the differences were statistically significant $p=0.0220$ ). Whereas, the mutated CYP2D6*4 allele was significantly more often present among the patients with dermatitis herpetiformis than in the control group (42.8\%, 24.4\%, respectively, $p=0.0351)$. The relative risk of developing dermatitis herpetiformis expressed by the odds ratio was 2-fold higher in subjects in whom the presence of the CYP2D6*4 allele (OR $=2.3 ; p=0.0351)$ was identified.

The relative risk of developing bullous skin diseases, including pemphigoid, pemphigus, and dermatitis herpetiformis, expressed by the OR was found to be higher for carriers of the mutated CYP2D6*3 allele (1.8, 2.6, and 2.3 , respectively). The risk, however, appeared to be statistically insignificant ( $p=0.6366, p=0.3884, p=0.9873$ ).

\section{Discussion}

In the process of drug biotransformation, a significant role has been attributed to cytochrome P-450 enzymes (CYP). The CYP enzymes show genetic polymorphism that leads to structural changes in coded proteins and modification of their activity. Differences in metabolic processes induced as a result of the presence of mutated alleles explain individual variability of responses to the same drug. They may also contribute to mutagenic and carcinogenic alterations in DNA and activation of toxic substances, thus initiating various pathogenic processes in the human organism.

Etiopathogenesis of numerous skin diseases has not been elucidated to date. The contemporary knowledge related to this subject reveals the presence of numerous factors affecting their development and suggests the role of genetic and environmental factors. Nowadays more attention has been drawn to the involvement of cytochrome P-450 and its isoenzymes in the course of dermatological diseases.

The studies on CYP2D6 polymorphism carried out among patients suffering from atopic diseases and psoriasis suggest an increase in the prevalence of the diseases in extensive metabolizers.

Recently, the CYP2D6 oxidation polymorphism has been observed to have an impact on the prevalence of atopic diseases, particularly bronchial asthma and allergic rhinitis. Nearly a 3-fold higher relative risk of development (OR $=2.81$ ) of these diseases was reported in EM subjects and almost 2-fold higher relative risk $(O R=1.85)$ of the extensive CYP2D6 genotype [44].

In the investigations carried out among patients suffering from psoriasis a more frequent prevalence of the disease has been stated in extensive metabolizers. The relative risk of psoriasis development was higher $(O R=1.22)$ in EM subjects as compared to subjects with intermediate and poor CYP2D6 genotype [45].

In recent years, the interest of researchers has been drawn to the role of oxidation polymorphism in autoimmune diseases such as systemic lupus erythematosus (SLE) and systemic sclerosis (SSc) [31-36]. Since both systemic as well as environmental factors have been shown to be involved in pathogenesis of these diseases, it is likely that polymorphisms of xenobiotic metabolizing enzymes including the polymorphism of CYP2D6 isoenzyme are associated with the immune system dysfunction that contributes to the development of autoimmune diseases.

The first reports on the relationship between the CYP2D6 genotype oxidation and autoimmune diseases were published in the 1990s.

The studies carried out among the patients with SLE demonstrated that the prevalence of CYP2D $6^{\star} 4$ allele was considerably higher in comparison to the control group (28.3\% and $18.6 \%$, respectively). However, the difference was not statistically significant. The CYP2D6*4 allele was observed to occur more often in SLE patients in whom renal or hematological lesions had been observed [35].

Polymorphism of CYP2D6 in SLE patients was also the subject of the study by Kortunay et al. The authors did not indicate any differences in the prevalence of particular genotypes in the group of SLE patients and in controls [36].

Similarly, Skrętkowicz et al. revealed that CYP2D6 polymorphism was not a risk factor for developing SLE. A slightly higher percentage of PM individuals (13.3\%) among SLE patients was observed as compared to healthy people (9.3\%); however, the differences were statistical- 
ly insignificant. Moreover, no statistically significant differences in CYP2D6 genotypes determining slow and fast oxidation in both groups studied were found [31]. Although the results of these studies show that the CYP2D6 oxidation polymorphism is not a risk factor for SLE development, the determination of the CYP2D6 genotype is of great clinical importance. Systemic lupus erythematosus is a multi-organ disease and there is often a need for administering drugs belonging to different therapeutic groups, including those that metabolize with the involvement of CYP2D6. The results of therapy with drugs undergoing oxidation are less satisfactory in extensive metabolizers as compared to poor metabolizers. High activity of CYP2D6 enzyme does not allow to reach therapeutic concentration of the drug, while low CYP2D6 enzyme activity and the associated slow metabolism increase the risk of adverse actions after administering a standard dose. Thus, the study of oxidation polymorphism may contribute to efficient and safe therapy.

There are scarce reports in the available literature presenting the influence of the polymorphism of cytochrome P-450 enzymes on the risk of SSc, which is characterized by a chronic autoimmune inflammatory process.

The study performed in the group of patients with SSC showed a statistically significant correlation between the prevalence of particular CYP2D6 genotypes and the risk of SSc. The relative risk of SSc development expressed by the odds ratio was over 5 -fold higher for carriers of the CYP2D6* $1 /$ CYP2D $6^{\star} 4$ genotype $(\mathrm{OR}=4.8, p<0.001)$ determining the extensive metabolism and 3 -fold higher for carriers of the mutated CYP2D6*4 allele $(O R=2.6$; $p=0.0002$ ) [32]. The results of the research suggest that the CYP2D6 oxidation polymorphism can be considered a risk factor for SSc development.

In our own studies concerning the effect of polymorphism in gene coding CYP2D6 enzyme on the prevalence of bullous skin diseases including pemphigoid, pemphigus, and dermatitis herpetiformis (DH), statistically significant correlations between the prevalence of the CYP2D6 genotype and the risk of DH development were observed. The relative risk of DH expressed by the OR was significantly higher for carriers of the CYP2D $6^{\star} 1 / C Y P 2 D 6^{\star} 4$ genotype $(\mathrm{OR}=4.23, p=0.0104)$. Moreover, a higher prevalence of the CYP2D6*4 allele was found in patients with $\mathrm{DH}$ as compared to the controls. The estimated relative risk of $\mathrm{DH}$ development expressed by the OR was 2-fold higher in CYP2D6*4 allele carriers $(\mathrm{OR}=2.3)$; this risk was statistically significant $(p=0.0351)$.

The results of the present study show that the CYP2D6 oxidation polymorphism cannot be considered a risk factor for development of pemphigoid and pemphigus, however it might have an impact on dermatitis herpetiformis. The research on the CYP2D6 oxidation polymorphism should be continued because there are no reports in the available literature presenting the effect of this polymorphism on the development of bullous skin diseases.
Numerous researchers have emphasized the role of polymorphism of xenobiotic metabolizing enzymes including the CYP2D6 oxidation polymorphism as a risk factor for various diseases. The interest has been more often drawn to autoimmune diseases due to their complex and not entirely elucidated etiology. The studies concerning polymorphism of xenobiotic metabolizing enzymes in the course of bullous skin diseases may contribute to greater efficacy and safety in pharmacotherapy and determine susceptibility to develop these diseases.

\section{Acknowledgments}

This work was supported by grants No. 503/8-01101/503-01 and No. 503/1-152-02/503-01 from the Medical University of Lodz.

\section{References}

1. Jabłońska S, Chorzelski T. Skin diseases. Wydawnictwo Lekarskie PZWL, Warsaw 2001.

2. Waszczykowska E, Wysoczańska K, Żebrowska A. The role of cytokines in the pathogenesis of autoimmune bullous skin disease. Alergia Astma Immunologia 2004; 9: 179-86.

3. Gornowicz-Porowska J, Seraszek-Jaros A, Kaczmarek E, et al. IgA autoantibodies to gliadin nonapeptides, tissue transglutaminase and epidermal transglutaminase are associated, but unrelated to neutrophil elastase expression in lesional skin in human dermatitis herpetiformis. Postep Derm Alergol 2012; 29: 233-9.

4. Cummins D, Mimouni DL, Tzu J, et al. Lichenoid paraneoplastic pemphigus in the absence of detectable antibodies. J Am Acad Dermatol 2007; 56: 153-9.

5. Erkiert-Polguj A, Żebrowska A, Wągrowska-Danilewicz M, et al. Expression of selected pro-apoptotic proteins in pemphigoid. Postep Derm Alergol 2011; 28: 6-14.

6. Feldman M, Brennan FM, Maini RN. Rheumatoid arthritis. Cell 1996; 85: 307-10.

7. Fong PH, Chan HL. Systemic lupus erythematosus with pemphigus vulgaris. Arch Dermatol 1985; 121: 26-7.

8. Korman NJ, Stanley JR, Woodley DT. Coexistence of pemphigus foliaceus and bullous pemphigoid. Arch Dermatol 1991; 127: 387-90.

9. Kubisz-Grek E, Kurhańska-Flisykowska A. A clinical case of pemphigus vulgaris. Dental Forum 2008; 36: 97-101.

10. Abenavoli L, Proietti L, Leggio L, et al. Cutaneous manifestations in celiac disease. World J Gastroenterol 2006; 12: 843-52.

11. Reunala T. Dermatitis herpetiformis. Clin Dermatol 2001; 19 : 728-36.

12. Czarnobilska E, Dyga W, Krzystyniak W, et al. Influence of environment exposures on the frequency of contact allergies in children and adolescents. Ann Agric Environ Med 2012; 19: 11-6.

13. Arlett CM, Black CM, Briggs DC, Stevens CO, Welsh KI. Telomere reduction in scleroderma patients, a possible cause of chromosomal instability. Br J Rheumatol 1996; 635: 732-7.

14. Guillam MT, Pédrono G, Le Bouquin S, et al. Chronic respiratory symptoms of poultry farmers and model-based estimates of long-term dust exposure. Ann Agric Environ Med 2013; 20: 307-11. 
15. Jenerowicz D, Silny W, Dańczak-Pazdrowska A, et al. Environmental factors and allergic diseases. Ann Agric Environ Med 2012; 19: 475-81.

16. Rychlik-Sych M, Skrętkowicz J. Drug metabolism. Farm Po 2008; 64: 51-60

17. Jaskuła-Sztul R. Polymorphism in detoxifying enzymes. Post Bioch 2005; 46: 50-9.

18. Skrętkowicz J, Rychlik-Sych M. Genetic cytochrome P-450 polymorphism. Farm Pol 2008; 64: 61-72.

19. Zanger UM, Raimundo S, Eichelbaum M. Cytochrome P450 2D6: overview and update on pharmacology, genetics, biochemistry. Naunyn Schmiedebergs Arch Pharmacol 2004; 369: 23-37.

20. Gawrońska-Szklarz B, Serwatowski P, Grodzki T, et al. Polymorphism of CYP2D6 and GSTM1 genes and susceptibility to lung cancer. Probl Ter Monitor 1998; 9: 158-64.

21. Gołąb-Janowska M, Honczarenko K, Gawrońska-Szklarz B, Potemkowski A. CYP2D6 gene polymorphism as a probable risk factor for Alzheimer's disease and Parkinson's disease with dementia. Neurol Neurochir Pol 2007; 41: 113-21.

22. Łapiński Ł, Wiela-Hojeńska A, Ganczarski G, et al. Clinical significance of oxidation and acetylation genetic polymorphism in pathogenesis of acute myeloblastic leukemia. Probl Ter Monitor 2006; 17: 11-9.

23. Mellick GD. CYP450, genetic and Parkinson's disease: gene $x$ environment interactions hold the key. J Neural Transm Suppl 2006; 70: 159-65.

24. Milejski P, Orzechowska-Juzwenko K, Niewiński P, et al. Clinical significance of oxidation and acetylation genetic polymorphism in patients with hyperthyreosis. Endokrynol Pol 2006; 6: 605-11.

25. Niewiński P. Clinical significance of sparteine oxidation and sulfadimidine acetylation polymorphism as indices of genetically determined metabolism of drugs and xenobiotics in patients with genito-urinary neoplasms. Probl Ter Monitor 1997; 8: 21-8.

26. D'Cruz D. Autoimmune diseases associated with drugs, chemicals and environmental factors. Toxicology Letters 2000; 112-113: 421-32.

27. Rychlik-Sych M, Skrętkowicz J, Gawrońska-Szklarz B, et al. Acetylation genotype and phenotype in patients with systemic lupus erythematosus. Pharmacol Rep 2006; 58: 22-9.

28. Skrętkowicz K, Skrętkowicz J, Gawrońska-Szklarz B, et al. Lack of association between arylamine $\mathrm{N}$-acetyltransferase 2 (NAT2) polymorphism and systemic sclerosis. Eur J Clin Pharmacol 2005; 60: 773-8.

29. May DG, Black CM, Olsen NJ, et al. Scleroderma is associated with differences in individual routes of drug metabolism, a study with dapsone, debrisoquine, and mephenytoin. Clin Pharmacol Ther 1990; 48: 286-95.

30. Sanchez E, Sabio JM, Callejas J, et al. Association study of genetic variants of proinflammatory chemokine and cytokine genes in systemic lupus erythematosus. BMC Med Genet 2006; 7: 48-55.

31. Skretkowicz J, Barańska M, Kaczorowska A, RychlikSych M. Genetic polymorphisms of CYP2D6 in patients with systemic lupus erythematosus. Arch Med Sci 2011; 7: 864-9.

32. Skrętkowicz J, Barańska M, Rychlik-Sych M. Genetic polymorphisms of CYP2D6 oxidation in patients with systemic sclerosis. Eur J Clin Pharmacol 2009; 65: 971-6.

33. von Schmiedeberg S, Fritsche E, Ronnau AC, et al. Polymorphisms of the xenobiotic-metabolizing enzymes CYP1A1 and NAT-2 in systemic sclerosis and lupus erythematosus. Adv Exp Med Biol 1999; 455: 145-52.
34. Yen JH, Chen ChJ, Tsai WC, et al. Cytochrome P450 and manganese superoxide dismutase genes polymorphisms in systemic lupus erythematosus. Immunol Lett 2003; 90: 19-24.

35. Sabbagh N, Marez D, Queyrel V, et al. Genetic analysis of cytochrome P450 CYP2D6 polymorphism in patients with systemic lupus erythematosus. Pharmacogenetics 1998; 8: 191-4.

36. Kortunay S, Bozkurt A, Bathum L, et al. CYP2D6 polymorphism in systemic lupus erythematosus patients. Eur J Clin Pharmacol 1999; 55: 21-5.

37. Bezier M, Reguiai Z, Vitry F, et al. Thiopurine S-methyltransferase genotype analysis in autoimmune bullous diseases. Eur J Dermatol 2008; 18: 512-7.

38. Oender K, Lanschuetzer CM, Laimer M, et al. Introducing a fast and simple PCR-RFLP analysis for the detection of mutant thiopurine S-methyltransferase alleles TPMT*3A and TPMT*3C. J Eur Acad Dermatol Venerol 2006; 20: 396-400.

39. Chrzanowska M, Kurzawski M, Droździk M, et al. Thiopurine S-methyltransferase phenotype-genotype correlation in hemodialyzed patients. Pharmacol Rep 2006; 58: 973-8.

40. Rychlik-Sych M, Skrętkowicz-Szarmach K, GawrońskaSzklarz B, et al. N-acetyltransferase 2 polymorphism in some autoimmune diseases. Probl Ter Monitor 2006; 17: 239-44.

41. Genc A, Gunasti S, Tuli A, et al. Thiopurine S-methyltransferase gene polymorphism in pemphigus vulgaris. Postep Derm Alergol 2012; 29: 25-9.

42. Smith CA, Gough AC, Leigh PN, et al. Debrisoquine hydroxylase gene polymorphism and susceptibility to Parkinson's disease. Lancet 1992; 6: 1375-7.

43. Gustincich S, Manfioletti G, Del Sal G, et al. A fast method for high-quality genomic DNA extraction from whole human blood. Biotechniques 1991; 1: 298-300.

44. Niewiński P, Patkowski J, Orzechowska-Juzwenko K, et al. CYP2D6 Phenotype versus genotype and the potential risk of allergic diseases. Adv Clin Exp Med 2005; 14: 1175-80.

45. Czarnecka A, Niewiński P, Orzechowska-Juzwenko K, et al. CYP2D6 oxidation genotype as a risk factor in development of psoriasis. Derm Klin 2005; 7: 25-7. 